AN. MED. InTERNA (Madrid) Vol. 18, N. $^{\circ} 7$, pp. 381-388, 2001

\title{
Objetivos y estrategia terapéutica en la diabetes mellitus tipo 2
}

\author{
J. Ma CALVO ROMERO, E. Ma LIMA RODRÍGUEZ*
}

Servicio de Medicina Interna. *Unidad Docente de Medicina Familiar y Comunitaria. Hospital Regional Universitario Infanta Cristina. Badajoz.

\author{
OBJECTIVES AND THERAPEUTIC STRATEGY IN TYPE 2 DIABETES \\ MELLITUS
}

\begin{abstract}
RESUMEN
El United Kingdom Prospective Diabetes Study (UKPDS) ha demostrado definitivamente que los diabéticos tipo 2 se benefician del control riguroso de la glucemia, ya que disminuye el riesgo de desarrollar complicaciones microvasculares. Los objetivos terapéuticos en la diabetes mellitus (DM) tipo 2 se han modificado con la finalidad de reducir el riesgo de estas complicaciones. No obstante, un tratamiento agresivo puede ser desastroso en pacientes que ya tienen complicaciones microvasculares y/o un riesgo aumentado de hipoglucemias inadvertidas, y tampoco sería aconsejable en pacientes mayores o con una corta esperanza de vida. Los fármacos disponibles para el tratamiento de la DM tipo 2 ofrecen muchas opciones para conseguir estos objetivos terapéuticos, debiendo tener siempre en cuenta las características individuales de cada paciente. En este artículo revisamos los objetivos en el control metabólico de la DM tipo 2 y sus fundamentos, y describimos brevemente la estrategia terapéutica recomendada para alcanzar estos objetivos, con atención especial a los nuevos antidiabéticos orales (repaglinida y tiazolidindionas)
\end{abstract}

PALABRAS CLAVE: Diabetes mellitus tipo 2. Control metabólico. Tratamiento. Antidabéticos orales.

\begin{abstract}
United Kingdom Prospective Diabetes Study (UKPDS) has demons trated definitively that patients with type 2 diabetes mellitus (DM) benefit from intensive blood glucose control, because it diminishes the risk to develop microvascular complications. The therapeutic targets in the type 2 DM have been modified in order to reduce the risk of these complica tions. However, aggressive treatment may be disastrous for patients with microvascular complications and/or an increased risk of hypoglycemic unawareness, and neither it would be advised in older patients or with short life expectancy. The available drugs for treatment of type 2 DM offer many options for achieving these therapeutic targets, based on the need of the individual patient. In this job we review the targets in the metabolic control of type 2 DM and their backgrounds, and we describe briefly the therapeutic strategy recommended for reaching these targets, with special attention to the new oral antidiabetic agents (repaglinide and thiazolidinediones).
\end{abstract}

KEY WORDS: Type 2 diabetes mellitus. Metabolic control. Treatment. Oral antidiabetic agents.

Calvo Romero JM", Lima Rodríguez EMa . Objetivos y estrategia terapéutica en la diabetes mellitus tipo 2. An Med Interna (Madrid) 2001; 18:381-388.

\section{INTRODUCCIÓN}

La prevalencia de la diabetes mellitus (DM) tipo 2 ha aumentado en las dos últimas décadas, debido probablemente al menos en parte a cambios en el hábito alimentario y a la mayor prevalencia en la población de la obesidad y de la inactividad física $(1,2)$. En España y en Europa se estima que la prevalencia de la DM es del 4\%, y que la DM tipo 2 es unas 10 veces más frecuente que la DM tipo 1 (1). Los pacientes con DM tipo 2 tienen una morbilidad y una mortalidad aumentadas debido a complicaciones microvasculares (retinopatía, nefropatía y neuropatía) y macrovasculares (cardiopatía isquémica, ictus y enfermedad vascular periférica) (3). La DM es una de la causas más frecuentes de ceguera en países desarrollados (3), la nefropatía diabética es la causa más frecuente de insuficiencia renal crónica terminal en países desarrollados (4), y la neuropatía periférica y autonómica ocurre en el 50$60 \%$ de los pacientes con DM tipo 2 (3). Los pacientes diabéticos tipo 2 tienen un riesgo de cardiopatía isquémica y de ictus aumentado en 2-4 veces (5). Estos datos dan idea de la importancia de la prevención de la complicaciones de la DM.

En los últimos años hemos conocido información de indudable interés sobre el riesgo de las complicaciones microvasculares y macrovasculares en la DM tipo 2. En este trabajo

Trabajo aceptado: 1 de Octubre de 2000

Correspondencia: J.Mª Calvo Romero. Héroes de Cascorro 9, $3^{\circ}$ A. 06004 Badajoz. 
revisamos los objetivos deseables en el control metabólico de la DM tipo 2 y sus fundamentos, y describimos brevemente la estrategia terapéutica recomendada para alcanzar dichos objetivos, con atención especial a los nuevos antidiabéticos orales (repaglinida y tiazolidindionas).

\section{FUNDAMENTOS}

En 1997 un comité de expertos revisó los criterios diagnósticos de la DM tomando como referencia los parámetros que se asocian a un riesgo aumentado de complicaciones (6). Estos criterios son: glucemia basal $\geq 126 \mathrm{mg} / \mathrm{dl}$, glucemia $\geq 200 \mathrm{mg} / \mathrm{dl}$ a las 2 horas de un test de sobrecarga oral de glucosa (SOG), y/o glucemia $\geq 200 \mathrm{mg} / \mathrm{dl}$ con síntomas clásicos de diabetes. Estos nuevos criterios evitan la discrepancia existente entre los antiguos criterios de glucemia basal $\geq 140$ $\mathrm{mg} / \mathrm{dl}$ y glucemia $\geq 200 \mathrm{mg} / \mathrm{dl}$ a las 2 horas de un test de SOG. Una glucemia basal $\geq 140 \mathrm{mg} / \mathrm{dl}$ supone un mayor grado de hiperglucemia que una glucemia $\geq 200 \mathrm{mg} / \mathrm{dl}$ a las 2 horas de un test de SOG (7). Una glucemia basal $\geq 126 \mathrm{mg} / \mathrm{dl}$ y una glucemia $\geq 200 \mathrm{mg} / \mathrm{dl}$ a las 2 horas de un test de SOG reflejan un grado similar de hiperglucemia y se asocian a un riesgo similar de desarrollar complicaciones microvasculares como la retinopatía diabética $(8,9)$. La prevalencia de complicaciones macrovasculares también está fuertemente relacionada con el nivel de glucemia basal y el nivel de glucemia a las 2 horas de un test de SOG (10-12).

\section{COMPLICACIONES MICROVASCULARES}

En 1993 se publicó el Diabetes Control and Complications Trial (DCCT), que demostró que la mejoría del control glucémico en los diabéticos tipo 1 con tratamiento intensivo con insulina disminuye el riesgo de desarrollar complicaciones microvasculares (13). Un pequeño estudio realizado en un grupo de 110 pacientes japoneses no obesos con diabetes tipo 2 seguidos durante 6 años obtuvo unos resultados con insulinoterapia intensiva similares a los del DCCT (14). No obstante, ningún estudio prospectivo y a largo plazo con un gran número de pacientes había demostrado que un control glucémico riguroso disminuyera la ocurrencia de complicaciones microvasculares en los diabéticos tipo 2. La mayoría de expertos, sin embargo, había concluido que los resultados del DCCT eran también aplicables a los diabéticos tipo 2 (15). En 1998 se publicó el United Kingdom Prospective Diabetes Study (UKPDS), un gran estudio prospectivo que intentaba determinar si el tramiento intensivo en los diabéticos tipo 2 prevenía el desarrollo de las complicaciones a largo plazo de la DM (16). Se comparó un grupo de 2.729 diabéticos tipo 2 con tratamiento intensivo con una sulfonilurea o con insulina (objetivo terapéutico: glucemia basal $<108 \mathrm{mg} / \mathrm{dl}$ ) con un grupo de 1.138 diabéticos tipo 2 con tratamiento dietético convencional (objetivos terapéuticos: glucemia basal $<270 \mathrm{mg} / \mathrm{dl}$ y ausencia de síntomas de hiperglucemia). En el grupo de tratamiento intensivo, a los pacientes que recibían una sulfonilurea se les añadió metformina o se pasó a insulinoterapia si no se consiguió el objetivo terapéutico. El tiempo medio de seguimiento fue 10 años. La hemoglobina glucosilada (HbA1c) fue sólo moderadamente menor en el grupo de tratamiento intensivo (7\% vs. $7,9 \%, \mathrm{p}<0,001)$. Hubo una reducción del $25 \%$ del riesgo de complicaciones microvasculares en el grupo de tratamiento intensivo. El UKPDS también estudió otro grupo de 342 diabéticos tipo 2 obesos con tratamiento intensivo con metformina (17). En este grupo se comprobó una reducción del 29\% del riesgo de complicaciones microvasculares. El UKPDS ha demostrado que el desarrollo de complicaciones microvasculares en los diabéticos tipo 2 se reduce con tratamiento intensivo con sulfonilureas, metformina y/o insulina, y que no hay un umbral para la reducción de la HbA1c, ya que a menor HbA1c menor es el riesgo de complicaciones microvasculares $(16,17)$.

\section{COMPLICACIONES MACROVASCULARES}

Las enfermedades cardiovasculares causan aproximadamente un $70 \%$ de la mortalidad en los pacientes con DM tipo $2(18,19)$. Un considerable número de estudios prospectivos en diabéticos tipo 2 ha demostrado una asociación entre el control glucémico y el desarrollo de eventos cardiovasculares (20-24). En un estudio de 1.370 diabéticos con un seguimiento de 10 años, un aumento de 1 punto en la HbA1c resultó en un aumento del $70 \%$ de retinopatía proliferativa, del $20 \%$ de proteinuria, pero sólo del $10 \%$ de eventos coronarios (20). Estos resultados sugieren que la hiperglucemia es un factor de riesgo mayor para el desarrollo de complicaciones microvasculares que para el desarrollo de complicaciones macrovasculares. No hay que olvidar que en muchos pacientes las complicaciones macrovasculares están ya presentes en el momento del diagnóstico de la DM tipo $2(18,19)$. La glucemia es un factor de riesgo cardiovascular incluso probablemente dentro de un rango situado por debajo del umbral diagnóstico de DM $(25,26)$. Por tanto, la glucemia sería un factor de riesgo cardiovascular continuo, de manera similar a la tensión arterial y a la colesterolemia $(25,26)$.

Diferentes estudios han intentado demostrar la utilidad de un control glucémico riguroso en la prevención de las complicaciones macrovasculares de la DM tipo 2, con resultados contradictorios. El University Group Diabetes Program estudió un amplio grupo de diabéticos tipo 2 durante un periodo de seguimiento medio de 12,5 años, sin que encontrara una reducción en la incidencia de eventos cardiovasculares en el grupo que recibió insulinoterapia intensiva (27). En este estudio los pacientes tratados con fenformina o tolbutamida tuvieron una mortalidad cardiovascular a los 7 años de seguimiento aumentada con respecto a los otros grupos, y este brazo del estudio fue suspendido $(28,29)$. No obstante, esta suspensión ha sido muy criticada ya que la mortalidad cardiovascular no aumentó en todos los centros del estudio, y ocurrió un número insuficiente de eventos cardiovasculares para obtener conclusiones definitivas. El Veterans Affairs Cooperative Study on Glycemic Control and Complications in Type 2 Diabetes estudió 153 varones diabéticos tipo 2 durante un seguimento medio de 27 meses, y tampoco encontró una reducción en la incidencia de eventos cardiovasculares en el grupo que recibió insulinoterapia intensiva, a pesar de que se consiguió una reducción de la HbA1c de 2 puntos, más bien al contrario hubo una tendencia sin alcanzar la significación estadística a una mayor frecuencia de eventos cardiovasculares en los pacientes con una HbA1c más baja (30). Por el contrario, en otro estudio que incluyó 620 diabéticos con infarto agudo de miocardio, la insulinoterapia intensiva se asoció a una reducción de la mortalidad a un año (31). En 
el UKPDS hubo una tendencia a un menor riesgo de infarto agudo de miocardio en el grupo de tratamiento intensivo, pero sin alcanzar significación estadística (16), aunque debe tenerse en cuenta que no hubo una gran diferencia en la $\mathrm{HbA} 1 \mathrm{c}$ entre los grupos de tratamiento intensivo y tratamiento convencional (7\% vs. $7,9 \%$ ). El tratamiento intensivo con metformina sí redujo el riesgo de desarrollar complicaciones macrovasculares en un grupo de diabéticos tipo 2 obesos estudiados en el UKPDS, con una reducción del 39\% del riesgo de infarto agudo de miocardio y del $41 \%$ del riesgo de ictus (17). A la vista de estos datos, son necesarios estudios adicionales para aclarar definitivamente el papel del control glucémico riguroso de la DM tipo 2 y de los diferentes fármacos disponibles en la prevención de las complicaciones macrovasculares.

\section{HIPERINSULINEMIA Y RESISTENCIA A LA INSULINA}

La resistencia a la insulina juega un papel etiopatogénico fundamental sobre todo en los pacientes con DM tipo 2 obesos (32). La DM tipo 2 forma parte en muchos casos de un síndrome metabólico complejo, denominado síndrome X o síndrome de resistencia a la insulina, que se caracteriza por la presencia de hiperinsulinemia, dislipemia, hipertensión arterial, obesidad, anomalías de la coagulación, microalbuminuria y ateroesclerois acelerada (33-35). No obstante, la relación causal entre insulino-resistencia/hiperinsulinemia y aterosclerosis/enfermedades cardiovasculares es tema de controversia. La resistencia a la insulina conduce a un aumento de la insulina endógena (32). Este aumento de la insulina endógena podría jugar un papel etiopatogénico en el desarrollo de la aterosclerosis por diversos mecanismos. La insulina podría estimular la proliferación de macrófagos y del músculo liso vascular y la agregabilidad plaquetaria, y disminuir la producción de prostaciclina por el endotelio vascular y la actividad del activador del plasminógeno (36). Muchos autores creen que la hiperinsulinemia puede ser un factor de riesgo cardiovascular (34-37). Un estudio realizado en Finlandia en 1.059 varones de mediana edad sin evidencia de enfermedad cardiovascular objetivó, tras un seguimiento de hasta 9,5 años, que la incidencia de muerte de causa cardiovascular y/o infarto agudo de miocardio no fatal era significativamente mayor en los individuos con mayores concentraciones plasmáticas de insulina, y un anális multivariante mostró que la concentración de insulina plasmática tras sobrecarga de glucosa era un predictor independiente de enfermedad cardiovascular (38-39).
En el Paris Prospective Study, que incluyó 7.434 varones de mediana edad sin enfermedad cardiovascular seguidos durante una media de 11 años, las concentraciones de insulina plasmática basal y tras sobrecarga de glucosa fueron factores de riesgo independientes para muerte de causa cardiovascular $(40,41)$.

Otro estudio australiano de 3.390 participantes varones y mujeres seguidos durante 13 años encontró una asociación entre enfermedad cardiovascular y la concentración de insulina plasmática tras sobrecarga de glucosa en varones entre los 60 y 69 años, pero no en mujeres ni en varones más jóvenes $(42,43)$. En otros estudios epidemiológicos en pacientes diabéticos, la hiperinsulinemia también parece ser un factor de riesgo de enfermedad cardiovascular $(44,45)$. Los estudios epidemiológicos mencionados proveen cierta evidencia de la asociación entre hiperinsulinemia y enfermedad cardiovascular. Esta asociación podría conllevar que la administración de insulina o fármacos que aumentan la secreción de insulina tuvieran un efecto perjudicial al aumentar el riesgo cardiovascular, hipótesis que sólo puede ser confirmada o rechazada definitivamente con la realización de estudios prospectivos con los diferentes fármacos. Por otro lado, en un estudio reciente la insulinoterapia intensiva en pacientes con DM tipo 2 se asoció a una mejoría del perfil lipídico, pero también a una elevación transitoria del fibrinógeno plasmático, con el consiguiente efecto teórico potencial trombógeno (46).

\section{OBJETIVOS TERAPÉUTICOS}

Los objetivos terapeúticos en la DM tipo 2 se han modificado con el fin de reducir el riesgo de complicaciones microvasculares. Los objetivos terapeúticos recomendados por la American Diabetes Association (ADA) se describen en la tabla I (47). Hay que tener presente que un tratamiento agresivo puede ser desastroso en pacientes que ya tienen complicaciones microvasculares y/o un riesgo aumentado de hipoglucemias inadvertidas. Tampoco sería aconsejable una terapia intensiva en diabéticos tipo 2 mayores o con una corta esperanza de vida (48).

La Veterans Health Administration (VHA) ha propuesto unas recomendaciones, estableciendo como objetivo terapéutico unos niveles más altos de $\mathrm{HbA} 1 \mathrm{c}$ en pacientes mayores de 75 años, pacientes con una corta esperanza de vida, con problemas de cumplimiento del tratamiento y/o con déficits intelectuales (48). La VHA establece el objetivo terapéutico de una $\mathrm{HbA} 1 \mathrm{c} £ 7 \%$ en diabéticos tipo 2 con una esperanza de vida mayor de 15 años en ausencia de complicaciones microvasculares o con una esperanza de vida de más de 10 años en presencia de enfermedad microvascular leve-moderada.

\section{TABLA I}

O BJETIVOS TERAPÉUTICOS EN LA DIABETES M ELLITUS TIPO 2 (AM ERICAN DIABETES ASSO CIATION)

\begin{tabular}{lccc}
\hline & No diabético & O bjetivo terapéutico & Recomendación de modificar tratamiento \\
\hline Glucemia preprandial & $<110 \mathrm{mg} / \mathrm{dl}$ & $80-120 \mathrm{mg} / \mathrm{dl}$ & $<80 \mathrm{mg} / \mathrm{dl} \mathrm{ó}>140 \mathrm{mg} / \mathrm{dl}$ \\
Glucemia al acostarse & $<120 \mathrm{mg} / \mathrm{dl}$ & $100-140 \mathrm{mg} / \mathrm{dl}$ & $<100 \mathrm{mg} / \mathrm{dl}$ ó $>160 \mathrm{mg} / \mathrm{dl}$ \\
Hemoglobina glucosilada & $<6 \%$ & $<7 \%$ & $>8 \%$ \\
\hline
\end{tabular}


En pacientes con una esperanza de vida de 5-15 años en ausencia de enfermedad microvascular o con una esperanza de vida de 5-10 años con enfermedad microvascular, la VHA recomienda conseguir una $\mathrm{HbA} 1 \mathrm{c£} \mathrm{8 \%}$. En diabéticos tipo 2 con una esperanza de vida inferior a los 5 años con o sin enfermedad microvascular, la VHA considera aceptable una HbA1c£ 9\%.

Los objetivos terapéuticos siempre deberán individualizarse teniendo en cuenta las características del paciente, especialmente en ancianos en los que el único objetivo del tratamiento puede ser el evitar los síntomas de hiperglucemia y el desarrollo de las complicaciones agudas de la DM.

\section{ESTRATEGIA TERAPÉUTICA}

El primer paso en la prevención de las complicaciones de la DM tipo 2 es el diagnóstico precoz de la enfermedad. La ADA recomienda la determinación de la glucemia en los individuos con factores de riesgo para el desarrollo de DM tipo 2, tales como historia familiar, obesidad, edad superior a 45 años, tolerancia alterada a la glucosa previa, hipertensión arterial, dislipemia, historia de diabetes gestacional o mujeres con hijos de más de $4 \mathrm{~kg}$ de peso al nacer (49). En los pacientes diabéticos debe prestarse gran atención a otros factores de riesgo cardiovascular como la dislipemia, la hipertensión arterial, la obesidad y el sedentarismo. Estos factores de riesgo cardiovascular coexisten con frecuencia con la DM tipo 2, muchas veces constituyendo el mencionado síndrome X (3335). Por ejemplo, el UKPDS demostró que la mejoría del control de la hipertensión arterial disminuye no sólo el riesgo de desarrollar complicaciones macrovasculares sino también el riesgo de complicaciones microvasculares en la DM tipo 2 (50). Otros estudios han demostrado que el tratamiento intensivo de la hipertensión arterial y de la hipercolesterolemia contribuye en los diabéticos tipo 2 a la prevención de las complicaciones cardiovasculares (51-54).

La ADA recomienda iniciar tratamiento farmacológico en la DM tipo 2 si la glucemia basal es igual o superior a $140 \mathrm{mg} / \mathrm{dl}$, la glucemia postprandial es igual o superior a $160 \mathrm{mg} / \mathrm{dl}$ y/o la HbA1c es igual o superior al 8\% (47). Probablemente a la vista de los resultados del UKPDS, el tratamiento farmacológico ya debería iniciarse con una $\mathrm{HbA} 1 \mathrm{c}$ del $7 \%$, ya que el mantenimiento de valores del 6-7\% se asocia a una reducción significativa del riesgo de complicaciones microvasculares $(16,17)$.

El arsenal terapéutico en la DM tipo 2 incluye la educación diabetológica, el tratamiento dietético, el ejercicio físico y el tratamiento farmacológico $(55,56)$. La pérdida de peso reduce la concentración de insulina plasmática y mejora la utilización periférica de glucosa, y junto con el ejercicio físico parece incrementar el número de receptores de insulina $(57,58)$. Los fármacos orales utilizados en el tratamiento de la DM tipo 2 pueden ser estimuladores de la secreción de insulina (sulfonilureas y repaglinida), sensibilizadores a la acción de la insulina con efecto reductor de la producción hepática de glucosa (metformina y tiazolidindionas), y enlentecedoresreductores de la absorción de glucosa (acarbosa y miglitol) $(55,56)$. En pacientes con DM tipo 2 que no consiguen un control metabólico adecuado con dieta y ejercicio físico debería iniciarse generalmente tratamiento con una sulfonilurea o con metformina, si no existen contraindicaciones $(55,56)$. La repaglinida también sería otra opción válida $(55,56)$. En monoterapia, las sulfonilureas, la metformina y la repaglinida tienen una eficacia similar en reducir la glucemia $(55,56)$. Debido a que la metformina favorece la pérdida de peso y mejora el perfil lipídico, es preferible en pacientes con DM tipo 2 obesos y/o con dislipemia (59). En los no obesos se podría iniciar tratamiento indistintamente con sulfonilureas, metformina o repaglinida. Las dosis en general pueden ser incrementadas en un periodo de 4-8 semanas hasta conseguir el objetivo terapéutico o alcanzar la dosis máxima $(55,56)$. Siempre se debe insistir en la dieta y en el ejercicio físico. Dada la alta prevalencia de enfermedad cardiovascular en los diabéticos tipo 2 y el probable aumento del riesgo cardiovascular que condiciona la hiperinsulinemia, parece recomendable diseñar una estrategia terapéutica que minimize la hiperinsulinemia.

Si la monoterapia falla en conseguir el control glucémico deseado, se recomienda asociar un segundo fármaco oral $(55,56)$. Otra opción sería añadir a la monoterapia una dosis de insulina intermedia antes de acostarse. Si la terapia combinada no consigue un control adecuado, existen 3 opciones: a) añadir insulina intermedia antes de acostarse, manteniendo uno o los 2 fármacos orales, b) cambiar a un régimen de insulina en monoterapia, o c) añadir un tercer agente oral $(55,56)$. Muchos autores defienden la ventaja de mantener los fármacos orales al añadir insulinoterapia, ya que ésto permite utilizar una menor dosis de insulina y conlleva un menor grado de hiperinsulinismo y una menor ganancia de peso (60-63).

\section{NUEVOS ANTIDIABÉTICOS ORALES}

En los últimos años el arsenal de fármacos orales eficaces en el tratamiento de la DM tipo 2 se ha ampliado, entre ellos merecen especial atención la repaglinida y las tiazolidindionas.

La repaglinida es un derivado del ácido benzoico, no perteneciente al grupo de las sulfonilureas, que estimula la secreción de insulina, mejorando la regulación de la glucemia prandial (64,65). Tiene una acción insulinotrópica (66), aumenta la secreción de insulina y de somatostatina, pero no causa una indeseable estimulación de la liberación de glucagón (67). Se desconoce si el efecto estimulador de la secreción de insulina de la repaglinida es aditivo al de las sulfonilureas. La repaglinida administrada por vía oral es rápidamente absorbida, inactivada en el hígado, y más del $90 \%$ se excreta en la bilis en forma de metabolitos inactivos $(68,69)$. Por su conducta farmacocinética, la repaglinida causa una rápida pero breve liberación de insulina.

Como se ha comentado previamente, la repaglinida utilizada en monoterapia tiene una eficacia similar a las sulfonilureas o a la metformina en reducir la glucemia basal y la HbA1c $(55,56,70-73)$. En diabéticos tipo 2 tratados previamente únicamente con dieta, la repaglinida reduce la glucemia basal en $50-60 \mathrm{mg} / \mathrm{dl}$ y la HbA1c en $1,7-1,9$ puntos $(72,73)$. La repaglinida no modifica de manera significativa los niveles plasmáticos de lípidos (72,73). El efecto de la repaglinida es aditivo al de la metformina. En diabéticos tipo 2 tratados previamente con metformina con un control metabólico inadecuado, la asociación de repaglinida consiguió reducir la glucemia basal $40 \mathrm{mg} / \mathrm{dl}$ y la HbA1c 1,4 puntos, siendo esta asociación superior a la monoterapia con metformina o repaglinida (74). 
La repaglinida ha sido comercializada recientemente en España. Las indicaciones de la repaglinida son similares a las de las sulfonilureas. Está indicada en el tratamiento de pacientes con DM tipo 2 en los que la dieta y el ejercico físico no consiguen un control adecuado de la glucemia $(55,56)$. La repaglinida puede utilizarse en alérgicos a sulfamidas, a diferencia de las sulfonilureas. Debido a su farmacocinética, la repaglinida no está contraindicada en pacientes con insuficiencia renal, al contrario que las sulfonilureas. En pacientes con hepatopatía, se recomienda una titulación de la dosis más lenta. La dosis de inicio de la repaglinida es $0,5 \mathrm{mg}$ vía oral, tomada 15 minutos antes de cada comida. En pacientes previamente tratados con otros antidiabéticos orales sin un control adecuado, se puede comenzar con una dosis de 1-2 mg antes de cada comida. La dosis máxima es de $4 \mathrm{mg}$ antes de cada comida en hasta un máximo de 4 comidas al día (16 $\mathrm{mg}$ /día). El aumento de la dosis se puede realizar semanalmente. Una dosis de $1 \mathrm{mg}$ de repaglinida tres veces al día produce un $90 \%$ del efecto máximo reductor de la glucemia (72). El tratamiento con una dosis de repaglinida antes de cada comida consigue un control glucémico similar independientemente del número de comidas realizadas al día, es bien tolerado, y permite un estilo de vida más flexible a los pacientes diabéticos tipo 2 (75). La hipoglucemia y el aumento de peso son los únicos efectos adversos que se presentan con mayor frecuencia en los pacientes tratados con repaglinida que en los que reciben placebo (76). Probablemente, la repaglinida causa menos hipoglucemias y de menor severidad que las sulfonilureas (70-73). El riesgo de hipoglucemia en los diabéticos tipo 2 tratados con repaglinida que omiten o retrasan una comida parece ser menor que en los tratados con sulfonilureas (77).

Las tiazolidindionas son un nuevo grupo de fármacos orales insulino-sensibilizadores eficaces en el tratamiento de la DM tipo 2 (78). Pertenecen a este grupo la troglitazona, la pioglitazona y la rosiglitazona. La rosiglitazona posiblemente será comercializada en breve en España. El efecto reductor de la glucemia de las tiazolidindionas se debe a la capacidad de estos fármacos de aumentar la sensibilidad a la insulina (78). Las tiazolidindionas mejoran la sensibilidad periférica a la insulina, especialmente en el músculo, y disminuyen la producción hepática de glucosa, sobre todo la gluconeogénesis (78). Igualmente reducen los niveles de insulina plasmática, y aumentan la sensibilidad a la insulina en el tejido adiposo (78).

La troglitazona, la primera tiazolidindiona utilizada en clínica, reduce la glucemia basal $25-45 \mathrm{mg} / \mathrm{dl}$ y la HbA1c $0,5-$ 0,9 puntos (79-82). La troglitazona es menos eficaz que las sulfonilureas o la metformina (83). Así por ejemplo, en un estudio en el que 76 pacientes con DM tipo 2 tratados previamente con gliburida cambiaron a tratamiento con $600 \mathrm{mg}$ diarios de troglitazona, tras un seguimiento de 52 semanas, la HbA1c aumentó 0,9 puntos (83). La troglitazona reduce los niveles de los triglicéridos plasmáticos en un 10-20\%, y aumenta los niveles de colesterol-HDL en un 5-10\% (79-81). Sin embargo, también aumenta los niveles plasmáticos de colesterol-LDL en un 10-15\% (79-81). La rosiglitazona y la pioglitazona tienen una eficacia similar a la troglitazona en reducir la glucemia basal y la HbA1c (84-87). La glucemia basal disminuyó $36 \mathrm{mg} / \mathrm{dl}$ en un grupo de diabéticos tipo 2 tratados con una dosis única diaria de $8 \mathrm{mg}$ de rosiglitazona (85). La rosiglitazona y la pioglitazona tienen efectos similares a la troglitazona sobre el perfil lipídico $(84,86,87)$.
Las tiazolidindionas pueden mejorar el perfil cardiovascular de los diabéticos tipo 2 (81,88). Algunos estudios han demostrado que las tiazolidindionas disminuyen la presión arterial moderadamente, fundamentalmente la diastólica, así como las resistencias periféricas por un mecanismo vasodilatador, y aumentan el índice cardíaco y el volumen latido $(81,88)$. Por ello, pueden contribuir al control de la hipertensión arterial en pacientes con DM tipo 2 (89). Las tiazolidindionas tienen propiedades anti-oxidantes, al inhibir la oxidación de las LDL, lo que podría impedir o retrasar el desarrollo de aterosclerosis (90).

Las tiazolidindionas estan indicadas en el tratamiento de la DM tipo 2 en aquellos casos en los que la dieta y el ejercico físico no consiguen un buen control metabólico $(55,56,78)$. Se recomiendan especialmente en pacientes obesos por la importancia etiopatogénica de la resistencia a la insulina en este grupo de diabéticos tipo $2(55,56,78)$. La asociación de troglitazona con sulfonilureas, metformina o insulina mejora el control metabólico de los pacientes con DM tipo 2 mal controlados con monoterapia (91-93). La asociación de rosiglitazona con sulfonilureas o metformina es igualmente eficaz $(94,95)$

La hepatotoxicidad es un efecto adverso importante de la troglitazona, por lo que ha sido retirada del mercado en Estados Unidos. El 1,9\% de los diabéticos tipo 2 tratados con troglitazona presentan una elevación de las transaminasas al menos 3 veces por encima del límite normal (96), en ocasiones con lesión hepática severa (97). El riesgo de hepatotoxicidad de la rosiglitazona parece ser mínimo (86), aunque recientemente se han publicado algunos casos aislados de hepatotoxicidad asociada al tratamiento con rosiglitazona $(98,99)$. No hay referencias en la literatura de hepatotoxicidad asociada a la pioglitazona (87). Los pacientes tratados con troglitazona experimentan un pequeño pero significativo aumento de peso $(79,80)$, que se debe fundamentalmente a un aumento de la grasa subcutánea más que a un aumento de la grasa visceral (100), ya que la grasa visceral puede incluso disminuir (101).

\section{CONCLUSIONES}

El UKPDS ha demostrado definitivamente que el tratamiento intensivo de la DM tipo 2 disminuye el riesgo de desarrollar complicaciones microvasculares. Los objetivos terapéuticos en la diabetes mellitus tipo 2 se han modificado con el fin de reducir estas complicaciones. No obstante, un tratamiento agresivo puede ser desastroso en pacientes que ya tienen complicaciones microvasculares y/o un riesgo aumentado de hipoglucemias inadvertidas, y tampoco sería aconsejable en pacientes mayores o con una corta esperanza de vida. Existen nuevos fármacos disponibles para el tratamiento de la DM tipo 2 que aumentan las opciones para conseguir un adecuado control metabólico, teniendo en cuenta siempre las características individuales de cada paciente. En la tabla II se comparan las características de los fármacos orales utilizados en el tratamiento de la diabetes mellitus tipo 2. En la prevención de la enfermedad macrovascular resulta fundamental el tratamiento de otros factores de riesgo cardiovascular frecuentemente asociados a la DM tipo 2. 
TABLA II

COM PARACIÓN DE LAS CARACTERÍSTICAS DE LO S FÁRM ACO S O RALES EN EL TRATAM IENTO DE LA DIABETES M ELLITUS TIPO 2

\begin{tabular}{|c|c|c|c|c|c|}
\hline & Sulfonilureas & M etformina & Acarbosa & Repaglinida & Rosiglitazona \\
\hline $\begin{array}{l}\text { M ecanismos de } \\
\text { acción }\end{array}$ & $\begin{array}{l}\text { Aumentan la } \\
\text { secreción de } \\
\text { insulina }\end{array}$ & $\begin{array}{l}\text { Disminuye la } \\
\text { producción hepática } \\
\text { de glucosa, aumenta } \\
\text { la sensibilidad a } \\
\text { la insulina }\end{array}$ & $\begin{array}{l}\text { Enlentece la } \\
\text { absorción } \\
\text { gastrointestinal } \\
\text { de glucosa }\end{array}$ & $\begin{array}{l}\text { Aumenta la } \\
\text { secreción de } \\
\text { insulina }\end{array}$ & $\begin{array}{l}\text { Aumenta la } \\
\text { sensibilidad a la } \\
\text { insulina, disminuye } \\
\text { la producción } \\
\text { hepática de glucosa }\end{array}$ \\
\hline $\begin{array}{l}\text { Disminución de la } \\
\text { glucemia basal (mg/dl) }\end{array}$ & $60-70$ & $60-70$ & $20-30$ & $50-60$ & $25-45$ \\
\hline
\end{tabular}

glucemia basal (mg/d )

Disminución de la

HbAlc (puntos de \%)

Triglicéridos

Colesterol-HDL

Colesterol-LDL

Peso corporal

Insulina plasmática

Efectos adversos

$1,5-2 \quad 1,5-2$

Sin efecto

Sin efecto

Sin efecto

Aumento

Aumento

Hipoglucemia

Disminución

Aumento ligero

Disminución

Disminución

Disminución

Digestivos,

acidosis láctica (rara)

Coste

M enor coste
2 veces el de las sulfonilureas
$0,7-1$

Disminución leve

Sin efecto

Sin efecto

Sin efecto

Sin efecto

Digestivos

2 veces el de las sulfonilureas
$1,7-1,9$

$0,5-0,9$

$\begin{array}{ll}\text { Sin efecto } & \text { Disminución } \\ \text { Sin efecto } & \text { Aumento } \\ \text { Sin efecto } & \text { Aumento } \\ \text { Aumento } & \text { Aumento } \\ \text { Aumento } & \text { Disminución } \\ \text { Hipoglucemia } & \text { Hepatotoxicidad? } \\ & \\ 2 \text { veces el de las } & 4 \text { veces el de las } \\ \text { sulfonilureas } & \text { sulfonilureas }\end{array}$

\section{Bibliografía}

1. Franch Nadal J, Álvarez Torices JC, Álvarez Guisasola F, et al. El diagnóstico de la diabetes mellitus en los estudios epidemiológicos. A Primaria $1991 ; 8$ : 465-470.

2. Helmrich SP, Ragland DR, Leung MP, Paffenbargen RS. Physical activity and reduced ocurrence of non-insulin dependent diabetes mellitus. $\mathrm{N}$ Engl J Med 1991; 325: 147-152.

3. Klein R. Hyperglycemia and microvascular and macrovascular disease in diabetes. Diabetes Care 1995; 18: 258-68.

4. Teutsch S, Newman J, Eggers P. The problem of diabetic renal failure in the United States: an overview. Am J Kidney Dis 1989; 13: 11-13.

5. Kannel WB, McGee DL. Diabetes and cardiovascular risk factors: the Framingham Study. Circulation 1979; 59: 8-13.

6. Report of the Expert Committee on the Diagnosis and Classification of Diabetes Mellitus. Diabetes Care 1997; 20: 1183-1197.

7. Harris MI, Hadden WC, Knowler WC, Bennet PH. Prevalence of diabetes and impaired glucose tolerance and plasma glucose levels in the US population aged 20-74 yr. Diabetes 1987; 36: 523-534.

8. McCance DR, Hanson RL, Charles MA, et al. Comparison of tests for glycated hemoglobin and fasting and two hour plasma glucose concentrations as diagnostic methods for diabetes. BMJ 1994; 308: 1323-1328.

9. Engelgau MM, Thompson TJ, Herman WH, et al. Comparison of fasting and 2-hour glucose and $\mathrm{HbA} 1 \mathrm{c}$ levels for diagnosing diabetes: diagnostic criteria and performance revisited. Diabetes Care 1997; 20: 785-791.

10. Charles MA, Balkau B, Vauzelle-Kervoeden E, Thibult N, Eschwege E. Revision of diagnostic criteria for diabetes. Lancet 1996; 348: 1657-1658.

11. Jackson CA, Yudkin JS, Forrest RD. A comparison of the relationships of the glucose tolerance test and the glycated haemoglobin assay with diabetic vascular disease in the community: the Islington Diabetes Survey. Diabetes Res Clin Pract 1992; 17: 111-123.

12. Beks PJ, Mackay AJC, de Neeling JND, de Vries H, Bouter LM, Heine RJ. Peripheral arterial disease in relation to glycaemic level in elderly Caucasian population: the Hoorn Study. Diabetologia 1995; 38: 86-96.

13. The Diabetes Control and Complications Trial Research Group (DCCT). The effect of intensive treatment of diabetes on the development and progression of long-term complications in insulin-dependent diabetes mellitus. N Engl J Med 1993; 329: 977-986.
14. Ohkubo Y, Kishibkawa H, Araki E, et al. Intensive insulin therapy prevents the progression of diabetic microvascular complications in Japanese patients with non-insulin-dependent diabetes mellitus: a randomized prospective 6-year study. Diabetes Res Clin Pract 1995; 28: 103-117.

15. Nathan DM. Inferences and implications. Do results from the Diabetes Control and Complications Trial apply in NIDDM? Diabetes Care 1995; 18: 251-257.

16. UK Prospective Diabetes Study Group. Intensive blood-glucose control with sulphonylureas or insulin compared with conventional treatment and risk of complications in patients with type 2 diabetes (UKPDS 33). Lancet $1998 ; 352$ : 837-853.

17. UK Prospective Diabetes Study Group. Effect of intensive blood-glucose control with metformin on complications in overweight patients with type 2 diabetes (UKPDS 34). Lancet 1998; 352: 854-865.

18. Pyörälä K, Laakso M, Uusitupa M. Diabetes and atherosclerosis: an epidemiologic view. Diabetes Metab Rev 1987; 3: 463-524.

19. Laakso M, Letho S. Epidemiology of macrovascular disease in diabetes. Diabetes Rev 1997; 5: 294-315.

20. Klein R. Hyperglycemia and microvascular and macrovascular disease in diabetes. Diabetes Care 1995; 18: 258-268.

21. Hanefeld M, Fisher S, Julius U, et al. Risk factors for myocardial infarction and death in newly detected NIDDM: the Diabetes Intervention Study: 11-year follow-up. Diabetologia 1996; 39: 1577-1583.

22. Letho S, Rönnemaa T, Pyörälä K, Laakso M. Predictors of stroke in middle-aged patients with non-insulin dependent diabetes. Stroke 1996; 27: 63-68.

23. Letho S, Rönnemaa T, Haffner SM, Pyörälä K, Kallio V, Laakso M. Dyslipemia and hyperglycemia predict coronary heart disease events in middle-aged patients with NIDDM. Diabetes 1997; 48: 1354-1359.

24. Turner RC, Millns H, Neil HAW, et al. Risk factors for coronary artery disease in non-insulin dependent diabetes mellitus. United Kingdom Prospective Diabetes Study (UKPDS 23). BMJ 1998; 316: 823-828.

25. Coutinho M, Gerstein HC, Wang Y, Yusuf S. The relationship between glucose and incident cardiovascular events: a metaregression analysis of published data from 20 studies of 95,783 individuals followed for 12.4 years. Diabetes Care 1999; 22: 233-240. 
26. Balkau B, Shipley M, Jarrett RJ, et al. High blood glucose concentrations is a risk factor for mortality in middle-aged nondiabetic men. Diabetes Care 1998; 21: 360-366.

27. University Group Diabetes Program. Effects of hypoglycemic agents on vascular complications in patients with adult-onset diabetes: final report. Diabetes 1982; 31: 1-81.

28. University Group Diabetes Program. A study of effects of hypoglycemic agents on vascular complications in patients with adult-onset diabetes. II. Mortality results. Diabetes 1970; 19: 789-830.

29. University Group Diabetes Program. A study of effects of hypoglycemic agents on vascular complications in patients with adult-onset diabetes. V. Evaluation of phenformin therapy. Diabetes 1975; 24 :65-184.

30. Abraira C, Colwell J, Nuttall F, et al. Cardiovascular events and correlates in the Veterans Affairs diabetes feasibility trial. Arch Intern Med 1997; 157: 181-188.

31. Malmberg K, Ryden L, Hamsten A, Herlitz J, Waldenström A, Wedel H, of the DIGAMI Study Group. Effects of insulin treatment on cause-specific one-year mortality and morbidity in diabetic patients with acute myocardial infarction. Eur Heart J 1996; 17: 1337-1344.

32. DeFronzo RA, Bonadonna RC, Ferrannini E. Pathogenesis of non-insulin dependent diabetes mellitus (NIDDM): a balanced overview. Diabetes Care 1992; 15: 318-68.

33. Ferrannini E, Haffner SM, Mitchell BD, Stern MP. Hyperinsulinaemia: the key of a cardiovascular and metabolic syndrome. Diabetologia 1991; 34: 416-422.

34. Reaven GM, Laws A. Insulin resistance, compensatory hyperinsulinemia, and coronary heart disease. Diabetologia 1994; 37: 948-952.

35. Reaven GM. Pathophysiology of insulin resistance in human disease. Physiol Rev 1995; 75: 473-486.

36. Stolar MW. Atherosclerosis in diabetes: the role of hyperinsulinemia. Metabolism 1988; 37: 1-9.

37. Stern MP. The insulin resistance syndrome: the controversy is dead, lonf live the controversy. Diabetologia 1994; 37: 956-958.

38. Pyörälä K. Relationship of glucose tolerance and plasma insulin to the incidence of coronary heart disease: results of two population studies in Finland. Diabetes Care 1979; 2: 131-141.

39. Pyörälä K, Savolainen E, Kaulola S, Haapakoski J. Plasma insulin as coroanry heart disease risk factor: relationship to other risk factors and predicitive value during 91/2 -year follow-up of the Helsinki policemen study population. Acta Med Scand 1985; 701: 38-52.

40. Fontbonne A, Tchobroutsky G, Eschwege E, et al. Coronary heart disease mortality risk: plasma insulin level is a more sensitive marker than hypertension or abnormal glucose tolerance in overweight males. Paris Prospective Study. Int J Obes 1988; 12: 557-565.

41. Fontbonne AM, Eschwege EM. Insulin and cardiovascular disease: Paris Prospective Study. Diabetes Care 1991; 14: 461-469.

42. Welborn TA, Wearne K. Coronary heart disease incidence and cardiovascular mortality in Busselton with reference to glucose and insulin concentrations. Diabetes Care 1979; 2: 154-160.

43. Cullen K, Stenhouse NS, Wearne KL, Welborn TA. Multiple regression analysis of risk factors for cardiovascular disease and cancer mortality in Busselton, Western Australia-12-year-study. J Chron Dis 1983; 36: 371-377.

44. Folsom AR, Syklo M, Stevens J, et al. A prospective study of coronary heart disease in relation to fasting insulin, glucose, and diabetes. Diabetes Care 1997; 20: 935-942.

45. Despres JP, Lamarche B, Mauriege P, et al. Hyperinsulinemia as an independent risk factor for ischemic heart disease. N Engl J Med 1996; 334: 952-957.

46. Emanuele N, Azad N, Abraira C, et al. Effect of intensive glycemic control on fibrinogen, lipids, and lipoproteins: Veterans Affairs Cooperative Study in Type II Diabetes Mellitus. Arch Intern Med 1998; 158: 2485-2490.

47. American Diabetes Association. Standards of medical care for patients with diabetes mellitus. Diabetes Care 1998; 21: 23-31.

48. Lardinois CK. Type 2 diabetes: glycemic targets and oral therapies for older patients. Geriatrics 1998; 53: 22-34.

49. American Diabetes Association. Screening for type 2 diabetes. Diabetes Care 1998; 21: 20-22.

50. Tight blood pressure control and risk of macrovascular and microvascular complications in type 2 diabetes: UKPDS 38. UK Prospective Diabetes Study. BMJ 1998; 317: 703-713.

51. Hansson L, Zanchetti A, Carruthers RG, et al. Effects of intensive bloodpressure lowering and low-dose aspirin in patients with hypertension: principal results of the Hypertension Optimal Treatment (HOT) randomised trial. Lancet 1998; 351: 1755-1762.
52. Pyörälä K, Pedersen TR, Kjekshus J, et al. Cholesterol lowering with simvastatin improves prognosis of diabetic patients with coronary heart disease. Diabetes Care 1997; 20: 614-620.

53. Sacks FM, Pfeffer MA, Moye LA, et al. The effect of pravastatin on coronary events after myocardial infarction in patients with average cholesterol levels. N Engl J Med 1996; 335: 1001-1009.

54. The Long-Term Intervention with Pravastatin in Ischaemic Disease (LIPID) Study Group. Prevention of cardiovascular events and death with pravastatin in patients with coronary heart disease and a broad range of initial cholesterol levels. N Engl J Med 1998; 339: 1349-1357.

55. Feinolas MN. Treatment of type 2 diabetes mellitus. Med Clin N Am 1998; 84: 757-90.

56. DeFronzo RA. Pharmacologic therapy for type 2 diabetes mellitus. An Intern Med 1999; 131: 281-303.

57. Kelly DE. Effects of weight loss on glucose homeostasis in NIDDM. Diabetes Rev 1995; 3: 366-376.

58. Sharma AM. Effects of nonpharmacological intervention on insulin sensitivity. J Cardiovasc Pharmacol 1992; 20: 27-34.

59. Bailey CJ, Path MRC, Turner RC. Metfomin. N Engl J Med 1996; 334: 574-579.

60. Yki-Järvinen H, Kauppila M, Kujansuu E, et al. Comparison of insulin regimens in patients with non-insulin-dependent diabetes mellitus. N Engl J Med 1992; 327: 1426-1433.

61. Yki-Järvinen H, Ryysy L, Nikkila K, Tulokas T, Venamo R, Heikkila M. Comparison of bedtime insulin regimens in patients with type 2 diabetes mellitus. A randomized, controlled trial. Ann Intern Med 1999; 130: 389396.

62. Johnson JL, Wolf SL, Kabadi UM. Efficacy of insulin and sulfonylurea combination therapy in type II diabetes. A meta-analysis of the randomized placebo-controlled trials. Arch Intern Med 1996; 156: 259-264.

63. Bergenstal R, Johnson M, Whipple D, et al. Advantages of adding metformin to multiple dose insulin therapy in type 2 diabetes. Diabetes 1998 ; 7: 89

64. Owens DR. Repaglinide-prandial glucose regulator: a new class of ora antidiabetic drugs. Diabet Med 1998; 15: 28-36.

65. Mooradian AD, Thurman JE. Drug therapy of postprandial hyperglycaemia. Drugs 1999; 57: 19-29.

66. Bakkali-Nadi A, Malaisse-Lagae F, Malaisse WJ. Insulinotropic action of meglitinide analogs: concentration-response relationship and nutrient dependency. Diabetes Res 1994; 27: 81-87.

67. Leclercq-Meyer V, Ladriere L, Fuhlendorff J, Malaisse WJ. Stimulation of insulin and somatostatin release by two meglitinides analogs. Endocrine 1997; 7: 311-317.

68. Hatorp V, Huang WC, Strange P. Repaglinide pharmacokinetics in healthy young adult and elderly subjects. Clin Ther 1999; 21: 702-710.

69. Hatorp V, Huang WC, Strange P. Pharmacokinetic profiles of repaglinide in elderly subjects with type 2 diabetes. J Clin Endocrinol Metab 1999; 84: $1475-1478$.

70. Marbury T, Huang WC, Strange P, Lebovitz H. Repaglinide versus glyburide: a one-year comparison trial. Diabetes Res Clin Pract 1999; 43: 155-166.

71. Wolffenbuttel BH, Landgraf R. A 1-year multicenter randomized doubleblind comparison of repaglinide and glyburide for the treatment of type 2 diabetes. Dutch and German Repaglinide Study Group. Diabetes Care 1999; 22: 463-467.

72. Schwartz SL, Goldberg RB, Strange P. Repaglinide in type 2 diabetes: a randomized, double blind, placebo-controlled, dose-response study. Repaglinide Study Group (Abstract). Diabetes 1998; 47: 98.

73. Goldberg RB, Einhorn D, Lucas CP, et al. A randomized placebo-controlled trial of repaglinide in the treatment of tye 2 diabetes. Diabetes Care 1998; 21: 1897-1903.

74. Moses R, Slobodniuk R, Boyages S, et al. Effect of repaglinide addition to metformin monotherapy on glycemic control in patients with type 2 diabetes. Diabetes Care 1999; 22: 119-124.

75. Damsbo P, Marbury TC, Hatorp V, Clauson P, Muller PG. Flexible prandial glucose regulation with repaglinide in patients with type 2 diabetes. Diabetes Res Clin Pract 1999; 45: 31-39.

76. Schatz H. Preclinical and clinical studies on safety and tolerability of repaglinide. Exp Clin Endocrinol Diabetes 1999; 107: 144-148.

77. Damsbo P, Clauson P, Marbury TC, Windfeld K. A double-blind randomized comparison of meal-related glycemic control by repaglinide and glyburide in well-controlled type 2 diabetic patients. Diabetes Care 1999; 22: 789-794.

78. Day C. Thiazolidinediones: a new class of antidiabetic drugs. Diabet Med 1999; 16: 179-192. 
79. Iwamoto Y, Kosaka K, Kuzuya T, Akanuma Y, Shigeta Y, Kaneko T. Effects of troglitazone: a new hypoglycemic agent in patients with NIDDM poorly controlled by diet therapy. Diabetes Care 1996; 19: 151-156.

80. Kumar S, Boulton AJ, Beck-Nielsen H, et al. Troglitazone, an insulin action enhancer, improves metabolic control in NIDDM patients. Troglitazone Study Group. Diabetologia 1996; 39: 701-709.

81. Ghazzi MN, Perez JE, Antonucci TK, et al. Cardiac and glycemic benefits of troglitazone treatment in NIDDM. The Troglitazone Study Group. Diabetes 1997; 46: 433-439.

82. Fonesca VA, Valiquet TR, Huang SM, Ghazzi MN, Whitcomb RW. Troglitazone montherapy improves glycemic control in patients with type 2 diabetes mellitus: a randomized, controlled study. The Troglitazone Study Group. J Clin Endocrinol Metab 1998; 83: 3169-3176.

83. Horton ES, Whitehouse F, Ghazzi MN, Venable TC, Whitcomb R. Troglitazone in combination with sulfonylurea restores glycemic control in patients with type 2 diabetes. The Troglitazone Study Group. Diabetes Care 1998; 21: 1462-1469.

84. Grossman S, Lessem J. Mechanisms and clinical effects of thiazolidinediones. Exp Opin Invest Drugs 1997; 6: 1025-1040.

85. Nolan JJ, Jones NP, Patwardhan R, Deacon LF. Rosiglitazone taken once daily provides effective glycaemic control in patients with Type 2 diabetes mellitus. Diabet Med 2000; 17: 287-284.

86. Goldstein BJ. Rosiglitazone. Int J Clin Pract 2000; 54: 333-337.

87. Gillies PS, Dunn CJ. Pioglitazone. Drugs 2000; 60: 333-345.

88. Sung BH, Izzo JL, Dandona P, Wilson MF. Vasodilatory effects of troglitazone improve blood pressure at rest and during mental stress in type 2 diabetes mellitus. Hypertension 1999; 34: 83-88.

89. Oghira T, Rakugi H, Idegami H, Mikami H, Masuo K. Enhancement of insulin sensitivity by troglitazone lowers blood pressure in diabetic hypertensives. Am J Hypertens 1995; 8: 316-320.

90. Noguchi N, Sakai H, Kato Y, et al. Inhibition of oxidation of low density lipoprotein by troglitazone. Atherosclerosis 1996; 123: 227-234.
91. Buysschaert M, Bobbioni E, Starkie M, Frith L. Troglitazone in combination with sulphonylurea improves glycaemic control in Type 2 diabetes patients inadequately controlled by sulphonylurea therapy alone. Troglitazone Study Group. Diabet Med 1999; 16: 147-153.

92. Inzuchi SE, Maggss DG, Spollet GR, et al. Efficacy and metabolic effects of metformin and troglitazone in type II diabetes mellitus. N Engl J Med 1998; 338: 867-872.

93. Schwartz S, Raskin P, Fonseca V, Graveline JF. Effect of troglitazone in insulin-treated patients with type II diabetes mellitus. N Engl J Med 1998; 338: 861-866.

94. Wolffenbuttel BH, Gomis R, Squatrito S, Jones NP, Patwardhan RN. Addition of low-dose rosiglitazone to sulphonylurea therapy improves glycaemic control in Type 2 diabetic patients. Diabet Med 2000; 17: 4047.

95. Fonseca V, Rosenstock J, Patwardhan R, Salzman A. Effect of metformin and rosiglitazone combination therapy in patients with type 2 diabetes mellitus: a randomized controlled trial. JAMA 2000; 283: 1695-1702.

96. Watkins PB, Whitcomb RW. Hepatic dysfunction associated with troglitazone. N Engl J Med 1998; 338: 916-917.

97. Gitlin N, Julie NL, Spurr CL, Lim KN, Juarbe HM. Two cases of severe clinical and histologic hepatotoxicity associated with troglitazone. Ann Intern Med 1998; 129: 36-38.

98. Forman LM, Simmons DA, Diamond RH. Hepatic failure in a patient taking rosiglitazone. Ann Intern Med 2000; 132: 118-121.

99. Al-Salman J, Arjomand H, Kemp DG, Mittal M. Hepatocellular injury in a patient receiving rosiglitazone. A case report. Ann Intern Med 2000; 132: 121-124.

100. Mori Y, Murakawa Y, Okada K, et al. Effect of troglitazone on body fat distribution in type 2 diabetic patients. Diabetes Care 1999; 22: 908-912

101. Kelly IE, Han TS, Walsh K, Lean ME. Effects of a thiazolidinedione compound on body fat distribution of patients with type 2 diabetes. Diabetes Care 1999; 22: 288-293. 